\title{
Lower is better: ezetimibe and PCSK9 inhibition join the mainstream of lipid-lowering therapy
}

\section{Cezary Wójcik ${ }^{1,2}$ \\ Assistant Professor, Department of Family Medicine, Oregon Health and Science University, Portland, USA \\ 2 Diplomate, American Board of Clinical Lipidology}

\section{Abstract}

The focus of 2013 cholesterol guidelines to prevent atherosclerotic cardiovascular disease (ASCVD) released by American College of Cardiology (ACC) and American Heart Association (AHA) is the administration of high intensity statin therapy to specific four groups of patients, which were found to benefit the most from such therapy. They no longer promote achieving specific LDL-C goals with a combination therapy involving statins and other drugs, as advocated by the former ATP-III guidelines as well as current guidelines of European Atherosclerosis Society, International Atherosclerosis Society or National Lipid Association. Such approach has been dictated by the strict reliance on randomized controlled trials as the only acceptable level of evidence. However, since publication of the 2013 ACC/AHA guidelines, cardiovascular benefits of ezetimibe added to statin therapy have been established. Moreover, the advent of PCSK9 inhibitors, providing a powerful supplement and/ or alternative to statin therapy, further complicates the therapeutic horizon in dyslipdiemias. It is very likely that a new set of ACC/AHA guidelines will be published in 2016, with a return of specific LDL-C and Non-HDL-C goals of therapy as well as integration of drugs other than statins. As the treatment of dyslipidemias becomes more complex, the need for the subspecialty of clinical lipidology to be officially recognized becomes more evident.

\section{Keywords}

Dyslipidemia; PCSK9 inhibitors; Ezetimibe; LDL; Clinical lipidology 


\section{Introduction}

Atherosclerotic cardiovascular disease (ASCVD) includes both coronary artery disease (CAD) as well as cerebrovascular disease (CVD)[1]. It remains one of the major killers worldwide [2]. Development of ASCVD is directly related to atherosclerotic damage caused over time (cholesterol-years) by various ApoB-containing lipoproteins, mostly LDL particles (atherogenic cholesterol). Therefore, lowering LDL-C by means of therapeutic lifestyle changes (TLCs) as well as pharmacotherapy is the principal approach to prevent ASCVD, both in primary and secondary preventions settings. In the world of LDL cholesterol, no doubt remains that "lower is better".

\section{Impact of the 2013 AHA/ACC Guidelines}

In 2013 the American Heart Association (AHA) and American College of Cardiology (ACC) released their cholesterol guidelines [1], which in the US have replaced 2001 ATP-III guidelines with 2004 update [3]. The 2013 ACC/AHA guidelines have dropped the LDL-C and Non-HDL-C targets of therapy, which until now, have been the base of all recommendations. Instead, they emphasized high intensity statin therapy in four "statin-benefit groups" identified using randomized clinical trials (RCTs). Those groups include:

1. patients with established ASCVD;

2. patients with LDL-C $\geq 190 \mathrm{mg} / \mathrm{dl}$;

3. patients aged 40-75 with DM and LDL-C 70-189 mg/dl;

4. patients aged 40-75 with 10-year ASCVD risk $\geq 7.5 \%$, as calculated by pooled cohort equations [1]. Treatment based on the new algorithm appears to better identify patients at higher ASCVD risk and increases cost-effectiveness of therapy when compared to the ATP-III recommendations with the 2004 update [4]. However, when using those guidelines some patients may be over-treated (those stratified as having ASCVD risk $\geq 7.5 \%$ using the pooled cohort equations), others may be undertreated (those at high $\mathrm{CV}$ risk, who have not achieved sufficient LDL-C lowering on statins alone). The authors of the new guidelines acknowledge that they have not provided a comprehensive algorithm to treat all lipid disorders, leaving ample space for additional interventions. The new guidelines also propose advanced lipid testing and coronary calcium imaging in selected patients with ASVD risk between 5\% and 7.5\% in order to identify additional individuals, which may benefit from statin therapy. It is also another myth, that the new guidelines do not advocate lipid testing once statin therapy is initiated. Lipid testing is recommended in order to check compliance and to evaluate whether patients are reaching the expected degree of LDL-C lowering ( $>50 \%$ for high intensity statin and $30-50 \%$ for medium intensity statin) [1]. Insurance companies and managed health care systems in the US have often implemented algorithms based on statin prescription alone, oversimplifying ASCVD prevention.

In sharp contrast to the US, the rest of the world still uses targets of therapy. They are included in the global recommendations for the management of dyslipidemia issued by the International Atherosclerosis Society [5] as well as in the European Atherosclerosis Society guidelines for management of dyslipidemias [6]. In the US, National Lipid Association has issued their own recommendations for the patient-centered management of dyslipidemia, which include goals of therapy with Non-HDL-C as the primary target [7].

One can argue, that in a way, the $2013 \mathrm{ACC} / \mathrm{AHA}$ guidelines still provide targets of therapy, which are no longer expressed as absolute LDL-C levels but as percentages in LDL-C reduction. Indeed, high intensity statin therapy advocated by the $2013 \mathrm{ACC} / \mathrm{AHA}$ guidelines is defined as causing an LDL-C drop $>50 \%$, medium intensity statin therapy is defined as causing a drop between $30-50 \%$ and low intensity statin therapy as causing a drop $<30 \%$ [1]. A hybrid approach is presented by 2012 guidelines from 
the Canadian Cardiovascular Society, where the goal of treatment is either to achieve LDL-C levels $\leq 2$ $\mathrm{mmol} / \mathrm{l}$ or $\geq 50 \%$ reduction of LDL-C [8].

The 2013 ACC/AHA guidelines relay strictly on randomized control trials (RCTs) and their metaanalysis, deliberately ignoring other lines of evidence, such as epidemiological data, subgroup analysis, genetic data or expert opinion stemming from basic research. Moreover, those guidelines emphasize statin therapy, marginalizing add-on drugs, such as ezetimibe or niacin, which lacked direct RCT evidence of their effectiveness in preventing ASCVD at the time of their publication [1]. This has significantly changed in the last two years, with new RCT data regarding ezetimibe and emerging evidence on inhibitors of proprotein convertase subtilisin/kexin type 9 (PCSK9). Niacin, on the other hand, has failed to show cardiovascular benefit as an add-on to statin therapy $[9,10]$. However, it remains a viable option for selected patients [11].

\section{Role of ezetimibe}

Ezetimibe leads to LDL-C lowering on average 12-25\% from baseline by a unique mechanism, through inhibition of the Niemann-Pick C1-like protein (NPC1LP), found on apical surface of enterocytes and thus inhibition of intestinal absorption of cholesterol as well as plant sterols [12,13]. Since the release of 2013 ACC/AHA guidelines, hard evidence has appeared, showing that addition of ezetimbe to simvastatin improves cardiovascular outcomes. Over a median follow up period of 7 years, the primary end-point consisting of combined incidence of CV death, MI, and stroke was significantly lower in the ezetimibe/simvastatin arm compared with the simvastatin arm (32.7\% vs. $34.7 \%$ respectively; number needed to treat $=50$ ). However, no significant differences were found in all-cause and CV mortality as well as need for coronary revascularization. This effect is presumably mediated by additional lowering LDL-C below previously recommended LDL-C targets [14]. Moreover, similar to statins, ezetimibe appears to have pleiotropic effects, leading to decrease in intravascular inflammation measured by HS-CRP levels [15]. Dual therapy with atorvastatin and ezetimibe leads to greater coronary plaque regression than with atorvastatin alone.

In addition to LDL-C lowering, ezetimibe causes a significant decrease in small dense LDL particles as well as remnant particles [16]. Intriguingly, the benefits of ezetimibe may stem from the fact that in contrast to statins it does not appear to upregulate PCSK9 [17]. Statins also upregulate the NPC1LP transporter, therefore increasing intestinal cholesterol absorption. Through NPC1LP inhibition, ezetimibe counters this unwanted side effect [12]. Addition of ezetimibe to the lowest dose of a given statin usually allows achieving LDL-C reduction similar to the highest dose of the same statin, while decreasing the chances for statin-associated side effects. Ezetimibe itself is well tolerated [13]. The main barrier to its use has been its relatively high cost and lack of reimbursement by many insurance carriers, who applied the statin-centric 2013 ACC/AHA guidelines in their payment rationale.

\section{Role of PCSK9 inhibitors}

The summer of 2015 has seen the approval in the US of two new lipid-lowering medications of a completely new class, called PCSK9 inhibitors (alirocumab [Praluent ${ }^{\mathrm{Tw}}$, marketed by Sanofi/Regeneron] and evolocumab [Repatha ${ }^{\mathrm{Tm}}$ marketed by Amgen]) [18] with a third agent of this class in clinical trials (bocozicumab, developed by Pfizer) [19]. Their development had an unprecedented pace leading from discovery of PCSK9 to approved drugs, which potentially will be used by millions.

The LDL receptor (LDL-R) has a central role in regulating LDL-C levels [20,21]. LDL-R binds LDL particles on hepatocyte surface, leading to the internalization of such complexes into the endosomal com- 
partment. Within endosomes, the LDL-R: LDL-particle complex undergoes dissociation. LDL particles are targeted for degradation mediated by lysosomal hydrolases, while LDL-R is recycled to the cellular surface [20]. This picture known well from medical student textbooks has been recently changed by the discovery that hepatocytes also produce PCSK9, a soluble protein released to the extracellular milieu. It eventually becomes associated with the LDL-R:LDL-particle complexes on the plasma membrane of hepatocytes. When internalized to endosomes, PCSK9 prevents the dissociation of LDL particles from LDL-R. As a consequence, the entire complex is degraded, downregulating LDL-R on hepatocyte surface and therefore decreasing the ability of removing LDL-particles from the circulation [22-24]. PCSK9 was originally discovered as a gain-of-function mutation leading to LDL elevation resembling classic familial hypercholesterolemia, but without associated mutations in LDL-R or ApoB. Hyperactive PCSK9 leads to overzealous LDL-R degradation, resulting in a poor clearance of LDL-particles and therefore elevated LDL-C levels and associated elevated CV risk [25,26]. Subsequently, several loss-offunction PCSK9 mutations have been described. In their case, lack of functional PCSK9 leads to overexpression of LDL-R, resulting in increased LDL-particle clearance and therefore decreased LDL-C ( 14-40\%) levels and associated decrease in CV risk ( 47-88\%) [24,27]. Other than having this beneficial effect, loss-of-function of PCSK9 did not cause any abnormalities in affected individuals. The latter finding has prompted the development of monoclonal antibodies able to bind and inactivate PCSK9. As mentioned above, two of such antibodies are already approved for clinical use (alirocumab and evolocumab) [18], while a third one (bococizumab) is in phase III clinical trials [19]. Evolocumab, alirocumab and bococizumab have achieved consistent and significant (60-80\%) reduction LDL-C levels either as monotherapy or when added to statin therapy [19,28-32]. Evolocumab has also been tested in patients with homozygous familial hypercholesterolemia, where it reduces LDL-C levels by $\sim 30 \%$, except in those with null LDL-R mutations [33]. As inferred from such spectacular LDL-C lowering, it can be hypothesized that PCSK9 inhibitors will lead to improved CV outcomes. Indeed, post hoc analysis of phase 3 studies of evolocumab and alirocumab have shown an additional 48-53\% reduction of CV events when added to statin therapy [34,35]. The CV benefits of those new drugs are going to be further investigated in large multicenter trials focused on evaluation of cardiovascular benefits [29,36,37].

Safety and tolerability of PCSK9 inhibitors appears to be similar to that of placebo, with rare injection site reactions and flu-like symptoms lasting several hours being the most common side effects [29,31]. The only concerning findings was the rate of neurocognitive adverse events which was found to be higher with evolocumab and alirocumab groups than with placebo in phase 3 trials [34,35].

An additional benefit of PCSK9 inhibitors is their ability to reduce Lp(a), an independent familial risk factor for ASCVD, which is not affected at all by statins [29-31,38]. Cholesterol associated with Lp(a) can contribute in some patients approximately 30-45\% to measured LDL-C levels as a percentage of its mass [38]. Evolocumab is able to reduce Lp(a) from 18 to $36 \%$ in a dose-dependent manner [30,31]. Alirocumab use also resulted in a significant reduction in $\operatorname{Lp}(\mathrm{a}) \sim 30 \%$ from baseline compared with placebo. Intriguingly, reductions in $\mathrm{Lp}$ (a) only weakly correlated with the magnitude of low-density lipoprotein cholesterol lowering [39].

\section{New guidelines on the horizon}

The landscape of ASCVD prevention is changing dramatically in front of our eyes. There is no doubt that statins will remain the cornerstone of therapy as their benefit, especially for the four groups of patients highlighted by the 2013 ACC/AHA guidelines, is well established in multiple RCTs [1]. Ezetimibe will likely become the recommended first line add-on on top of statins, leaving PCSK9 inhibitors as the last resource. 
Without set goals of therapy, it is hard to envision a rational algorithm for treatment escalation, especially one involving the addition of expensive but very powerful injectable drugs such as PCSK9 inhibitors. Regardless of the approach, the number of patients requiring those drugs may be very significant, if we consider statin-intolerance as an indication for their use. Most patients tolerate statins well, but up to $29 \%$ experience side effects (primarily muscle-related) contributing to diminished compliance or discontinuation of statin therapy and subsequent increase in cardiovascular risk [40]. However, statin intolerance if often very subjective, as it has been recently shown that up to $70 \%$ of patients previously characterized as "statin-intolerant" actually tolerate well $20 \mathrm{mg}$ of atorvastatin daily, if they are not aware that they are taking a statin [41].

In the new guidelines, not only the old LDL-C goals of therapy are likely to return, but also a new LDL$\mathrm{C}$ target of $\leq 40 \mathrm{mg} / \mathrm{dl}$ may emerge for the very high-risk patients. It is reasonable, that new guidelines will provide a hybrid approach between that of ATP-III and 2013 ACC/AHA guidelines, advocating lipid lowering therapy tailored to achieve either $\leq 70 \mathrm{mg} / \mathrm{dl} \mathrm{LDL}-\mathrm{C}$ levels $\mathrm{OR} \geq 50 \%$ reduction of LDLC, similar to the approach of 2012 CCS guidelines [8].

Moreover, rather than focusing solely on LDL-C, Non-HDL-C may become the main target of therapy, as it better approximates cardiovascular risk [7]. This is due to the fact that Non-HDL-C correlates better with levels of LDL-particles. The latter can be discordant with LDL-C levels in as many as 30\% of patients. There is also some degree of discordance between Non-HDL-C and LDL-particles, which may be also measured directly or by measurement of $A p o B$ levels, providing alternate targets for therapy $[42,43]$. However, their measurement is expensive and requires advanced testing, while Non-HDL-C can be directly calculated from standard non-fasting or fasting lipid panel. The use of Non-HDL-C as the primary target of therapy has been already advocated by the National Lipid Association [7]. Both Non-HDL-C and ApoB are alternate targets in the CCS guidelines [8].

\section{Questions for further research}

The causative relation between atherosclerosis and levels of atherogenic cholesterol allows to hypothesize, that the use of PCSK9 inhibitors will likely lead to significant reduction in ASCVD mortality and morbidity. Indeed, post hoc analysis from phase III trials have shown 48-53\% reduction of CV events when PSCK9 inhibitors are added to statin therapy [34,35], but full data are still lacking [44]. Several large cardiovascular outcome trials are under way to address this issue. The ODYSSEY Outcomes trial will test the effects of alirocumab on the combined endpoint of CHD death, MI, ischemic stroke, or hospitalization for unstable angina in 18,000 patients with a starting LDL-C $\geq 70 \mathrm{mg} / \mathrm{dl}$ randomized 1-16 weeks post-ACS who have been treated with statin therapy. ODYSSEY outcomes trial will be finalized by March of 2018 [36]. The FOURIER trial will test the effects of evolocumab on the combined end-point of CV death, MI, stroke, hospitalization for unstable angina or coronary revascularization in 22,500 patients with previous MI, stroke or peripheral arterial disease with a starting LDL-C $\geq 70 \mathrm{mg} /$ $\mathrm{dl}$ who have been treated with atorvastatin $20 \mathrm{mg}$ or higher or equivalent therapy. It will be finalized by December 2017 [29]. Finally, the SPIRE trials (SPIRE I and II) will test the effects of bococizumab on the combined endpoint of CV death, MI, stroke or urgent revascularization. SPIRE I will focus on 12,000 patients with LDL-C between 70 and $99 \mathrm{mg} / \mathrm{dl}$, while SPIRE II will focus on 6300 patients with LDL-C $\geq 100 \mathrm{mg} / \mathrm{dl}$, all of them on appropriate baseline lipid lowering therapy. The outcome of the SPIRE trials will be known by August 2017. The completion of those trials will provide ample evidence to include PCSK9 inhibitors in future guidelines. It will also address the potential safety concerns [44]. The cost of therapy with monoclonal antibodies for a common disease, such as dyslipidemia, will have a likely impact on insurance policies and reimbursement [37]. In an attempt to rationalize their use, their prescription may be relegated from primary care physicians and cardiologists to subspecialists in clinical lipidology. 


\section{The review in brief}

The novel approach of the 2013 ACC/AHA cholesterol guidelines to prevent ASCVD consisted in abandoning LDL-C goals of therapy and focusing on initiation on medium to high intensity statin therapy in groups of patients with high CV risk [1]. However, this approach is no longer consistent with emerging evidence of cardiovascular benefits of ezetimibe [14] and PCSK9 inhibitors [45]. There is an urgent need for new guidelines, which will integrate the rational use of this new class of agents. As our armamentarium of lipid lowering drugs grows and the landscape of cardiovascular prevention becomes more complicated, it is becoming clear that a population-based "one size fit all" solution to cardiovascular prevention may not be optimal. Personalization of lipid lowering therapy and its fitting to individualized $\mathrm{CV}$ risk requires significant expertise, heralding the imminent approval of clinical lipidology as the new medical subspecialty in the US.

\section{References}

1. Stone NJ, Robinson JG, Lichtenstein AH, et al.; American College of Cardiology/American Heart Association Task Force on Practice Guidelines. 2013 ACC/AHA guideline on the treatment of blood cholesterol to reduce atherosclerotic cardiovascular risk in adults: a report of the American College of Cardiology/American Heart Association Task Force on Practice Guidelines. J Am Coll Cardiol 2014; 63: 2889-934; http://dx.doi.org/10.1016/j.jacc.2013.11.002

2. Mendis S, Puska P, Norrving B, et al. Global atlas on cardiovascular disease prevention and control. World Health Organization in collaboration with the World Heart Federation and the World Stroke Organization: Geneva, 2011

3. Grundy SM, Cleeman JI, Merz CN, et al.; Coordinating Committee of the National Cholesterol Education Program. Implications of recent clinical trials for the National Cholesterol Education Program Adult Treatment Panel III Guidelines. J Am Coll Cardiol 2004; 44: 720-32; http://dx.doi. org/10.1016/j.jacc.2004.07.001

4. Jung CH, Lee MJ, Kang YM, et al. 2013 ACC/AHA versus 2004 NECP ATP III Guidelines in the Assignment of Statin Treatment in a Korean Population with Subclinical Coronary Atherosclerosis. PLoS One 2015; 10: e0137478; http://dx.doi.org/10.1371/journal.pone.0137478

5. Expert Dyslipidemia Panel of the International Atherosclerosis Society Panel, m. An International Atherosclerosis Society Position Paper: global recommendations for the management of dyslipidemia--full report. J Clin Lipidol 2014; 8: 29-60; http://dx.doi.org/10.1016/j.jacl.2013.12.005

6. Catapano AL, Reiner Z, De Backer G, et al. ESC/EAS Guidelines for the management of dyslipidaemias The Task Force for the management of dyslipidaemias of the European Society of Cardiology (ESC) and the European Atherosclerosis Society (EAS). Atherosclerosis 2011; 217: 3-46; http://dx.doi.org/10.1016/j.atherosclerosis.2011.06.028

7. Jacobson TA, Ito MK, Maki KC, et al. National Lipid Association recommendations for patientcentered management of dyslipidemia: part 1 - executive summary. J Clin Lipidol 2014; 8: 473-88; http://dx.doi.org/10.1016/j.jacl.2014.07.007

8. Anderson TJ, Grégoire J, Hegele RA, et al. 2012 update of the Canadian Cardiovascular Society guidelines for the diagnosis and treatment of dyslipidemia for the prevention of cardiovascular disease in the adult. Can J Cardiol 2013; 29: 151-67; http://dx.doi.org/10.1016/j.cjca.2012.11.032

9. Teo KK, Goldstein LB, Chaitman BR, et al.; AIM-HIGH Investigators. Extended-release niacin therapy and risk of ischemic stroke in patients with cardiovascular disease: the Atherothrombosis Intervention in Metabolic Syndrome with low HDL/High Triglycerides: Impact on Global Health Outcome (AIM-HIGH) trial. Stroke 2013; 44: 2688-93; http://dx.doi.org/10.1161/ STROKEAHA.113.001529 
10. HPS2-THRIVE Collaborative Group. HPS2-THRIVE randomized placebo-controlled trial in 25673 high-risk patients of ER niacin/laropiprant: trial design, pre-specified muscle and liver outcomes, and reasons for stopping study treatment. Eur Heart J 2013; 34: 1279-91; http://dx.doi. org/10.1093/eurheartj/eht055

11. Zeman M, Vecka M, Perlík F, et al. Niacin in the Treatment of Hyperlipidemias in Light of New Clinical Trials: Has Niacin Lost its Place? Med Sci Monit 2015; 21: 2156-62; http://dx.doi. org/10.12659/MSM.893619

12. Fazio, S. The role of PCSK9 in intestinal lipoprotein metabolism: synergism of statin and ezetimibe. Atheroscler 2015; 17: 23-6; http://dx.doi.org/10.1016/S1567-5688(15)50006-8

13. Phan BA, Dayspring TD, Toth PP. Ezetimibe therapy: mechanism of action and clinical update. Vasc Health Risk Manag 2012; 8: 415-27

14. Cannon CP, Blazing MA, Giugliano RP, et al.; IMPROVE-IT Investigators. Ezetimibe Added to Statin Therapy after Acute Coronary Syndromes. N Engl J Med 2015; 372: 2387-97; http://dx.doi. org/10.1056/NEJMoa1410489

15. Bohula EA, Giugliano RP, Cannon CP, et al. Achievement of Dual Low-Density Lipoprotein Cholesterol and High-Sensitivity C-Reactive Protein Targets More Frequent With the Addition of Ezetimibe to Simvastatin and Associated With Better Outcomes in IMPROVE-IT. Circulation 2015; 132: 1224-33; http://dx.doi.org/10.1161/CIRCULATIONAHA.115.018381

16. Sakamoto K, Kawamura M, Kohro T, et al.; RESEARCH Study Group. Effect of Ezetimibe on LDL-C Lowering and Atherogenic Lipoprotein Profiles in Type 2 Diabetic Patients Poorly Controlled by Statins. PLoS One 2015; 10: e0138332; http://dx.doi.org/10.1371/journal.pone.0138332

17. Miyoshi T, Nakamura K, Doi M, et al. Impact of Ezetimibe Alone or in Addition to a Statin on Plasma PCSK9 Concentrations in Patients with Type 2 Diabetes and Hypercholesterolemia: A Pilot Study. Am J Cardiovasc Drugs 2015; 15: 213-9; http://dx.doi.org/10.1007/s40256-015-0119-2

18. White CM. Therapeutic Potential and Critical Analysis of the PCSK9 Monoclonal Antibodies Evolocumab and Alirocumab. Ann Pharmacother 2015; 49: 1327-35; http://dx.doi. org/10.1177/1060028015608487

19. Ballantyne CM, Neutel J, Cropp A, et al. Results of bococizumab, a monoclonal antibody against proprotein convertase subtilisin/kexin type 9, from a randomized, placebo-controlled, doseranging study in statin-treated subjects with hypercholesterolemia. Am J Cardiol 2015; 115: 1212 21; http://dx.doi.org/10.1016/j.amjcard.2015.02.006

20. Goldstein JL, Brown MS. The LDL receptor. Arterioscler Thromb Vasc Biol 2009; 29: 431-8; http:// dx.doi.org/10.1161/ATVBAHA.108.179564

21. Brown MS, Goldstein JL. Receptor-mediated endocytosis: insights from the lipoprotein receptor system. Proc Natl Acad Sci USA 1979; 76: 3330-7; http://dx.doi.org/10.1073/pnas.76.7.3330

22. Qian YW, Schmidt RJ, Zhang Y, et al. Secreted PCSK9 downregulates low density lipoprotein receptor through receptor-mediated endocytosis. J Lipid Res 2007; 48: 1488-98; http://dx.doi. org/10.1194/jlr.M700071-JLR200

23. Horton JD1, Cohen JC, Hobbs HH. PCSK9: a convertase that coordinates LDL catabolism. J Lipid Res 2009; 50 Suppl: S172-7; http://dx.doi.org/10.1194/jlr.R800091-JLR200

24. Cohen J, Pertsemlidis A, Kotowski IK, et al. Low LDL cholesterol in individuals of African descent resulting from frequent nonsense mutations in PCSK9. Nat Genet 2005; 37: 161-5; http://dx.doi. org/10.1038/ng0305-328c

25. Abifadel M, Varret $M$, Rabès JP, et al. Mutations in PCSK9 cause autosomal dominant hypercholesterolemia. Nat Genet 2003; 34: 154-6; http://dx.doi.org/10.1038/ng1161

26. Allard D, Amsellem S, Abifadel M, et al. Novel mutations of the PCSK9 gene cause variable phenotype of autosomal dominant hypercholesterolemia. Hum Mutat 2005; 26: 497; http://dx.doi. org/10.1002/humu.9386 
27. Cohen JC, Boerwinkle E, Mosley TH Jr, et al. Sequence variations in PCSK9, low LDL, and protection against coronary heart disease. $N$ Engl J Med 2006; 354: 1264-72; http://dx.doi. org/10.1056/NEJMoa054013

28. Farnier M. An evaluation of alirocumab for the treatment of hypercholesterolemia. Expert Rev Cardiovasc Ther 2015; 13: 1307-23; http://dx.doi.org/10.1586/14779072.2015.1111759

29. Langslet G, Emery M, Wasserman SM. Evolocumab (AMG 145) for primary hypercholesterolemia. Expert Rev Cardiovasc Ther 2015; 13: 477-88; http://dx.doi.org/10.1586/14779072.2015.1030395

30. Cicero AF, Tartagni E, Ertek S. Efficacy and safety profile of evolocumab (AMG145), an injectable inhibitor of the proprotein convertase subtilisin/kexin type 9: the available clinical evidence. Expert Opin Biol Ther 2014; 14: 863-8; http://dx.doi.org/10.1517/14712598.2014.902929

31. Blom DJ, Hala T, Bolognese M, et al.; DESCARTES Investigators. A 52-week placebo-controlled trial of evolocumab in hyperlipidemia. N Engl J Med 2014; 370: 1809-19; http://dx.doi.org/10.1056/ NEJMoa1316222

32. Verbeek R, Stoekenbroek RM2, Hovingh GK. PCSK9 inhibitors: Novel therapeutic agents for the treatment of hypercholesterolemia. Eur J Pharmacol 2015; 763: 38-47; http://dx.doi.org/10.1016/j. ejphar.2015.03.099

33. Raal FJ, Honarpour N, Blom DJ, et al.; TESLA Investigators. Inhibition of PCSK9 with evolocumab in homozygous familial hypercholesterolaemia (TESLA Part B): a randomised, double-blind, placebo-controlled trial. Lancet 2015; 385: 341-50; http://dx.doi.org/10.1016/S01406736(14)61374-X

34. Hassan M. OSLER and ODYSSEY LONG TERM: PCSK9 inhibitors on the right track of reducing cardiovascular events. Glob Cardiol Sci Pract 2015; 2015: 20; http://dx.doi.org/10.5339/gcsp.2015.20

35. Robinson JG, Farnier M, Krempf M, et al.; ODYSSEY LONG TERM Investigators. Efficacy and safety of alirocumab in reducing lipids and cardiovascular events. N Engl J Med 2015; 372: 148999; http://dx.doi.org/10.1056/NEJMoa1501031

36. Schwartz GG, Bessac L, Berdan LG, et al. Effect of alirocumab, a monoclonal antibody to PCSK9, on long-term cardiovascular outcomes following acute coronary syndromes: rationale and design of the ODYSSEY outcomes trial. Am Heart J 2014; 168: 682-9; http://dx.doi.org/10.1016/j. ahj.2014.07.028

37. Schulman KA, Balu S, Reed SD. Specialty Pharmaceuticals for Hyperlipidemia--Impact on Insurance Premiums. N Engl J Med 2015; 373: 1591-3; http://dx.doi.org/10.1056/NEJMp1509863

38. Yeang C, Witztum JL, Tsimikas S. 'LDL-C' $=\mathrm{LDL}-\mathrm{C}+\mathrm{Lp}(\mathrm{a})-\mathrm{C}$ : implications of achieved ultra-low LDL-C levels in the proprotein convertase subtilisin/kexin type 9 era of potent LDL-C lowering. Curr Opin Lipidol 2015; 26: 169-78; http://dx.doi.org/10.1097/MOL.0000000000000171

39. Gaudet D, Kereiakes DJ, McKenney JM, et al. Effect of alirocumab, a monoclonal proprotein convertase subtilisin/kexin 9 antibody, on lipoprotein(a) concentrations (a pooled analysis of 150 mg every two weeks dosing from phase 2 trials). Am J Cardiol 2014; 114: 711-5; http://dx.doi. org/10.1016/j.amjcard.2014.05.060

40. Jacobson TA. NLA Task Force on Statin Safety--2014 update. J Clin Lipidol 2014; 8(3 Suppl): S1-4; http://dx.doi.org/10.1016/j.jacl.2014.03.003

41. Moriarty PM, Jacobson TA, Bruckert E, et al. Efficacy and safety of alirocumab, a monoclonal antibody to PCSK9, in statin-intolerant patients: design and rationale of ODYSSEY ALTERNATIVE, a randomized phase 3 trial. J Clin Lipidol 2014; 8: 554-61; http://dx.doi. org/10.1016/j.jacl.2014.09.007

42. Cromwell WC, Otvos JD, Keyes MJ, et al. LDL Particle Number and Risk of Future Cardiovascular Disease in the Framingham Offspring Study - Implications for LDL Management. J Clin Lipidol 2007; 1: 583-92; http://dx.doi.org/10.1016/j.jacl.2007.10.001 
43. Degoma EM, Davis MD, Dunbar RL, et al. Discordance between non-HDL-cholesterol and LDLparticle measurements: results from the Multi-Ethnic Study of Atherosclerosis. Atherosclerosis 2013; 229: 517-23; http://dx.doi.org/10.1016/j.atherosclerosis.2013.03.012

44. Doggrell SA, Lynch KA. Is there enough evidence with evolocumab and alirocumab (antibodies to proprotein convertase substilisin-kexin type, PCSK9) on cardiovascular outcomes to use them widely? Evaluation of Sabatine MS, Giugliano RP, Wiviott SD et al. Efficacy and safety of evolocumab in reducing lipids and cardiovascular events. N Engl J Med 2015;372:1500-1509, and Robinson JG, Farnier M, Krempf M et al. Efficacy and safety of alirocumab in reducing lipids and cardiovascular events. N Engl J Med 2015;372:1488-99. Expert Opin Biol Ther 2015; 15: 1671-5; http://dx.doi.org/10.1517/14712598.2015.1093109

45. Shapiro MD, Fazio S, Tavori H. Targeting PCSK9 for therapeutic gains. Curr Atheroscler Rep 2015; 17: 499; http://dx.doi.org/10.1007/s11883-015-0499-4 